\title{
A New Approach to Selectively Implement Control Flow Error Detection Techniques
}

\author{
Jens Vankeirsbilck, Jonas Van Waes, Hans Hallez and Jeroen Boydens
}

\begin{abstract}
Many software-implemented control flow error detection techniques have been proposed over the years. In an effort to reduce their overhead, recent research has focused on selective approaches. However, correctly applying these approaches can be difficult. This paper aims to address this concern and proposes a new approach. Our new approach is easier to implement and is applicable on any existing control flow error detection technique. To prove its validity, we apply our new approach to the Random Additive Control Flow Error Detection technique and perform fault injection experiments. The results show that the selective implementation has approximately the same error detection ratio with a decrease in execution time overhead.
\end{abstract}

\section{Introduction}

The reliability of embedded systems in ever harsher working environments is becoming more important. These systems are, however, more vulnerable to external disturbances ranging from high energy particles striking the hardware to electromagnetic interference and attackers $[13,4,14]$. These external disturbances introduce bit-flips in the systems hardware, which can cause invalid behavior such as erroneously controlling an actuator, corrupting data or corrupting the execution order of instructions.

The corruption of the execution order of instructions is also known as a control flow error (CFE). A CFE is a violation against the control flow graph (CFG) of the program. The CFG is a representation of the program, in which the program is divided into basic blocks and edges. A basic block is a sequence of consecutive instructions with exactly one entry and one exit point. An edge is an intentional path

Jens Vankeirsbilck, Jonas Van Waes, Hans Hallez, Jeroen Boydens

Department of Computer Science KU Leuven, Spoorwegstraat 12, 8200 Brugge, Belgium e-mail: \{jens.vankeirsbilck, jonas.vanwaes, hans.hallez, jeroen.boydens $\} @$ kuleuven.be 
between basic blocks. CFEs are typically partitioned into two categories: inter-block CFEs and intra-block CFEs. An inter-block CFE is an invalid jump through the program between two different basic blocks, while an intra-block CFE is an invalid jump within the same basic block. Both types of CFE can cause the affected program or system to halt, to crash or to provide erroneous output, potentially leading to hazardous situations.

To increase the reliability of embedded systems, several software-implemented CFE detection techniques, have been proposed $[10,11]$. In a previous comparative study, we developed a CFE detection technique called RACFED which has a higher detection ratio at a lower execution time overhead when compared to similar techniques [15]. The imposed execution time overhead, however, is still relatively high. Therefore, research has focused on selective CFE detection techniques [5, 9]. Selective CFE detection techniques only protect a selected part of the target program to reduce the imposed execution time overhead. Whilst reducing the execution time overhead, selective techniques are often difficult to implement correctly or are difficult to apply to other CFE detection techniques.

This paper aims to address this concern by proposing a new principle to selectively implement CFE detection techniques. Our new principle is relatively easy to implement and can be used on any CFE detection technique. To validate this principle, we apply it to our RACFED technique to create Selective RACFED or S-RACFED. The rest of this paper is organized as follows. We discuss more background and related work in Section 2. Next, we discuss S-RACFED and how to implement it in Section 3. Then, we validate S-RACFED and present the experiment setup and the results in Section 4 and Section 5, respectively. Conclusions are drawn in Section 6.

\section{Background and Related Work}

Past years, a multitude of CFE detection techniques has been proposed [12, 6, 1, $10,2,3,11]$. In order to detect CFEs, such techniques insert extra control variables, called signatures, and instructions into the target program at compile time. At run time, the added instructions recalculate these control variables and compare them to their expected compile-time value. A mismatch indicates that a CFE has been detected. In previous work, the authors performed a comparative study of these techniques and used the data to develop a new CFE detection technique called Random Additive Control Flow Error Detection (RACFED) [15]. When compared to similar techniques, RACFED shows to be the better technique since it detects more CFEs at a lower overhead.

The above mentioned techniques are full implementation techniques, i.e., they insert the control variable update and verification instructions in each basic block in the CFG. In recent years, selective implementations have been proposed, where the extra instructions are only inserted in certain selected basic blocks. One way to selectively apply CFE detection, is to simply ignore some basic blocks. This approach 
is taken by the S-SETA technique [5]. Within S-SETA, smaller basic blocks have no extra instructions inserted in order to reduce the imposed execution time overhead. While this drastically reduces the overhead, it also reduces the detection ratio, as CFEs that occur within the ignored basic blocks are possibly undetectable.

A second way to to selectively apply CFE detection, is to insert signature update instructions in each basic block, but to only insert signature verification instructions in selected basic blocks. Chielle et al. propose to only insert the verification instructions in larger basic blocks with their SETA-C technique [5]. Khudia et al. took another approach with their Abstract Control Signatures (ACS) technique [9]. They divide the basic blocks into regions and assign a specific signature to each region. Each basic block in a region has a signature update instruction inserted, while signature verification instructions are only inserted in basic blocks where the control flow changes from one region to another. Khudia et al. define their regions as a collection of basic blocks which has a single entry point but multiple exit points. Ideally, the regions should possess certain properties that help in minimizing the number of signature updates and verifications.

Although SETA-C and ACS show promise, implementing them can be difficult. SETA-C only inserts the verification instructions in larger basic blocks. For algorithms with little difference between the length in basic blocks, the decrease in execution time overhead can be limited. To gain the maximum decrease in execution time overhead when applying ACS, the division of the CFG into regions is critical. However, Khudia et al. provide little guidance on how to find this optimal division.

Therefore, we propose an easier and more consistent approach to selectively implement CFE detection techniques. We propose to only insert the signature verification instructions in return basic blocks. These are basic blocks which contain the return statement that exits out of the current function or program and returns control to the calling function or program. These basic blocks are the last basic block in each possible path through the target CFG and thus allow to detect all CFEs that occur within the CFG.

\section{Selective RACFED}

This section discusses how we applied the proposed selective implementation principle to our RACFED technique. First, we discuss how the principle transforms RACFED into S-RACFED with a high-level example. Next, we present the small change to be made to the compile-time process to implement RACFED to implement it selectively. 


\subsection{High-level example}

RACFED is our CFE detection technique that uses run-time signature updates to detect both inter- and intra-block CFEs. A high-level implementation of RACFED is shown on the left-hand side of Fig. 1. The instructions inserted by RACFED are indicated in bold and $S$ is the run-time signature. As can be seen, a signature update is inserted at the beginning of each basic block and after each non-jump original instruction. In RACFED, each basic block has a signature verification instruction that compares the run-time signature with the compile-time value. These are indicated as S != \#value: error (). When a mismatch between the run-time and compile-time value is detected, a call is made to the error-handler.

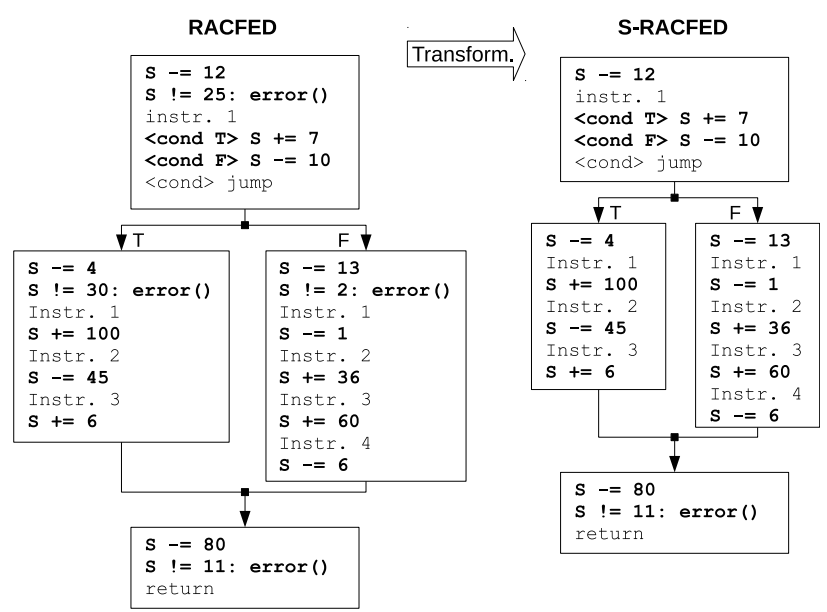

Fig. 1 Transformation from RACFED to S-RACFED.

Applying our proposed selective implementation approach transforms RACFED in S-RACFED. As shown in the right-hand side of Fig 1, only the bottom basic block has a signature verification instruction inserted because that is the return basic block. Since S-RACFED does not remove any signature update instructions, it should have the same CFE detection ratio as RACFED but with a lower execution time overhead.

\subsection{Change in Compile-time Process}

There are four steps in the compile-time process to implement RACFED:

1. First, all needed compile-time variables are assigned to all basic blocks.

2. Then, the instruction monitoring is implemented. These update instructions help to detect intra-block CFEs. 
3. Next, the first signature update and the signature verification instructions are inserted in each basic block.

4. Finally, the last signature update is inserted in each basic block.

To implement S-RACFED, the third step is slightly adjusted. The first signature update instruction is still inserted in each basic block. The signature verification, however, is only inserted in return basic blocks. Therefore, an extra check is inserted in the compile-time process that analyses whether or not the last instruction of the basic block is a return instruction. If so, only then the signature verification is inserted in the basic block.

\section{Experimental Setup}

To thoroughly validate the proposed approach, we performed fault injection experiments, measured the error detection cost and measured the error detection latency of S-RACFED and RACFED. This section discusses the setup of the performed experiments in detail. First, we present the four criteria we measured for S-RACFED. Next, we present the selected case studies and the selected target. Finally, we discuss the used fault injection process.

\subsection{Criteria}

To compare S-RACFED with RACFED, we measured four criteria: the effect of each error, the execution time overhead, the code size overhead and the error detection latency.

\subsubsection{Error Effects}

For every injected fault, we determined its effect. We grouped the effects in four categories:

- Detected (Det.): This percentage of faults was detected by the implemented countermeasure. In other words, this category is the desired result.

- Hardware Detection (HD): Many processors already have several internal fault handlers that are able to detect specific hardware faults, such as improper bus usage or memory access violations. This category represents the faults detected by such fault handler.

- Silent Data Corruption (SDC): These are the faults that were not detected by the implemented technique and caused the algorithm to produce a wrong result.

- No Effect (NE): Finally, this is the percentage of the faults that were not detected and did not affect the outcome of the target algorithm. 


\subsubsection{Error Detection Cost}

Software-implemented CFE detection techniques insert extra instructions into the code they need to protect. This means that the code size and the execution time of the protected code will be higher than that of the unprotected code. We measured both its code size and execution time and compare the results to the unprotected code. The code size is measured using the text output of the arm-none-eabi-size tool, when used on the .elf file of the compiled case study. The execution time is measured using a hardware timer of the target.

\subsubsection{Error Detection Latency}

Because the main difference between RACFED and S-RACFED is the placement of the verification instruction, we measured the error detection latency of both techniques. For every detected CFE, we counted the number of executed instructions before the CFE was detected.

\subsection{Case Studies}

The selected case studies are an implementation of the following algorithms: bit count (BC), bubble sort (BS), cycle redundancy check (CRC), cubic function solver (CU), Dijkstra's algorithm (DIJ), fast fourier transform (FFT), matrix multiplication (MM) and quick sort (QS). While the BS, MM and QS case studies use our own implementation, the other implementations were selected from MiBench version 1.0 [7].

With the selected case studies, a wide array of embedded application domains is covered. The BS, QS, MM and FFT case studies are also the typical applications used to validate error detection techniques in literature [12, 6, 1, 10, 2, 3, 11]. Furthermore, they also have varying basic block and edge distributions which assures a thorough validation of our approach. Some setup and validation code is required to launch the case studies and verify their results. However, only the function implementing the target algorithm will have (S-)RACFED applied and only that function will be subjected to CFEs.

\subsection{Target}

We executed the case studies on an ARM Cortex-M3, because it is an industryleading 32-bit processor used in many different embedded application domains.

For the CFE injection experiments, we used a simulated Cortex-M3 provided by the Imperas simulator [8]. The Imperas simulator is an instruction set simulator 
which allows to execute the target instructions at host (computer) speed, speeding up the fault injection experiments. The Imperas simulator supports ARM, MIPS, PowerPC and several other targets.

Because the Imperas simulator is an instruction accurate simulator and not a cycle accurate simulator, it is less suited to perform timing measurements. Therefore, we performed the execution time measurements on a physical target, the NXP LPC 1768. The NXP LPC 1768 is an ARM Cortex-M3 driven microcontroller running at $96 \mathrm{MHz}$, including $512 \mathrm{kB}$ FLASH and $32 \mathrm{kB}$ RAM.

\subsection{Fault Injection Process}

The used CFE injection process starts with an initialization phase that initializes variables and creates all possible CFEs. Those possible CFEs are constructed from the user-defined options disasmFile and range. The disasmFile holds the path to the disassembly file of the target program. This file is needed to know which program counter (PC) values are valid for the program. The range option indicates for which PC range the target program will be tested. The option allows to target a specific function (or functions) to be tested, instead of the entire program. All created possible CFEs are within this range.

Once the possible CFEs are created, the execution part of the fault injection process starts. This phase begins with performing a check to determine whether or not the previous loop injected a CFE. In case a CFE was injected, a new instance of the virtual platform is created and a handler to the processor is obtained. Next, the processor executes the number of steps indicated by execSteps variable. The execSteps variable indicates how far into the case study the fault injection testing has already progressed. When no CFE was injected, the processor of the current instance of the virtual platform is progressed one step further and the corresponding variables are updated.

Next, the current $\mathrm{PC}$ of the processor is read out and the loop variable $\mathrm{CFE}_{-} \mathrm{In}-$ jected is updated. The read PC value is compared to the user specified endAddress value. The endAddress is the PC value that indicates the end of the current fault injection experiment. When this value is reached, all desired CFEs have been injected and the experiment can thus stop. In our case, the endAddress is the return address of the function implementing the target algorithm. In other words, in our case the endAddress is the first PC value of the verification part of the case study.

The next step in the algorithm is to determine whether or not the read out PC falls within the user specified range. When the PC is not in that range, a new CFE attempt is started and the execution phase restarts. In case the PC does fall within the range, its according CFE possibilities are extracted from all possibilities that were created at the start of the algorithm.

Finally, the process analyses whether for the current PC a new CFE must be injected. This is determined by comparing the current wanted possibility index (possIndex) with the number of possibilities for this PC. If a CFE must be 
injected, it is injected, analysed for its effect on the target program, the according variables are updated and the current instance of the virtual platform is deleted. Then, the execution part of the fault injection restarts.

When no CFE must be injected, a final check is executed. This final check determines whether or not the current PC must be removed from the range of allowed PC values. It does this by checking if the total amount of injected CFEs exceeds the limit provided by the user via the minCFEs option. When the total amount of injected CFEs exceeds the limit, the PC is removed. The minCFEs option is mainly used to break the process out of case studies that have loops with a lot of iterations. In this case, the loop iterations will inject the same CFEs until the minCFEs limit is reached. Once reached, the $\mathrm{PC}$ values part of the loop will be removed from the allowed PC range, causing the fault injection experiment to skip them and test the remainder of the target program.

\section{Results}

This section represents the results of the performed experiments per defined criterion.

\subsection{Fault Injection Results}

For each of the selected case studies, we defined five datasets and we subjected each combination of case study, dataset and implemented technique to fault injection experiments. The results are shown in Fig. 2 and are the averages for the five datasets. The green bar indicates the percentage of CFEs that was detected by the implemented technique (Det), the red bar indicates the percentage of CFEs that was not detected and caused a wrong result (SDC), and gray bar shows the percentage of CFEs that were either detected by the microcontroller itself or that were not detected and did not corrupt the result of the case study (HD + NE).

As shown, the impact of implementing RACFED selectively changes from case study to case study. For the CU, DIJ, and QS case studies, the SDC ratio of SRACFED is approximately the same as when RACFED is implemented. For the DIJ case study, S-RACFED even has a higher error detection ratio. A minor negative impact on the SDC ratio is measured for the BS and FFT case studies. For these two case studies, the SDC ratio increases with one or two percent. The FFT case study also reports a much lower error detection ratio with S-RACFED implemented, but the undetected errors are mainly covered by either the hardware or the application resilience. However, for the $\mathrm{BC}, \mathrm{CRC}$ and $\mathrm{MM}$ case studies, the chart illustrates that the selective implementation has a major negative impact on both the error detection ratio and the SDC ratio. For these case studies, the SDC ratio increases with five to ten percent, resulting in an intolerable SDC ratio between ten and eighteen percent. 


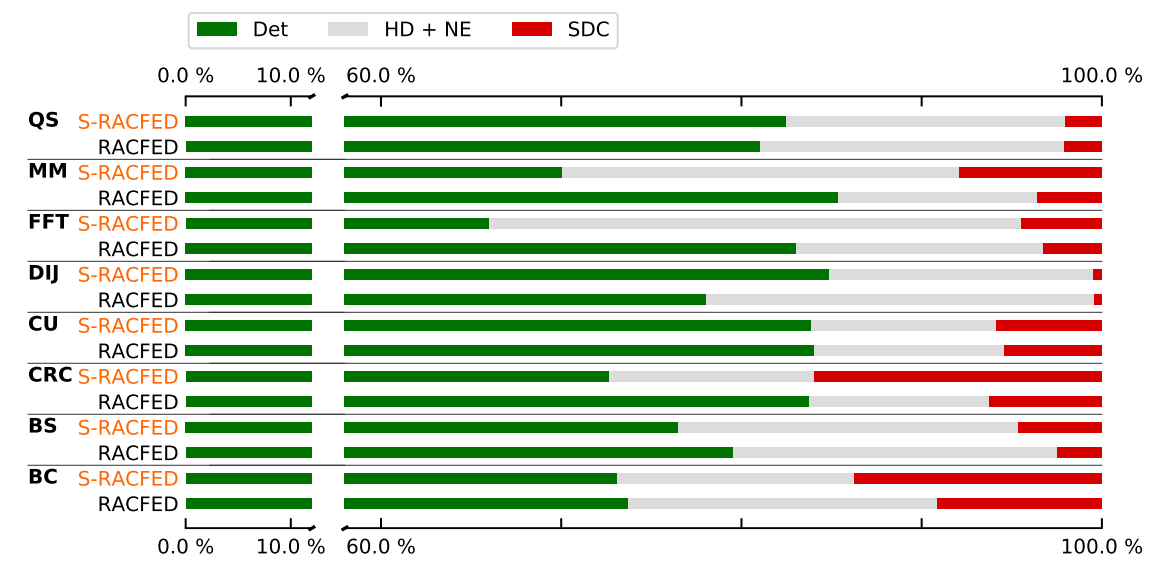

Fig. 2 Bar chart presenting the results of the fault injection experiments.

Regarding the CRC and MM case studies, this increase is coupled with a significant decrease in error detection ratio.

Although little to no difference between the fault injection results of RACFED and S-RACFED were expected, the results indicate that, depending on the case study, large differences are possible. Especially for the smaller case studies, i.e. $\mathrm{BC}$ and CRC, bigger differences were noted. The data shows that when S-RACFED is implemented, more single-bit CFE resulted in premature undetected exits out of the algorithm, increasing the SDC ratio.

\subsection{Error Detection Cost}

The error detection cost of the techniques is shown in Fig. 3. The dark-blue bars show the execution time of each technique compared to the unprotected code, while the light-blue bars present the code size of each technique.

Here, the chart displays the expected results, with S-RACFED imposing less execution time overhead and code size overhead. Again, depending on the case study, the reduction in overhead can be larger. On average, the execution time with SRACFED implemented is $\times 1.66$ that of the unprotected code. In contrast, the execution time with RACFED implemented is $\times 1.87$ compared to the unprotected code, on average. The reduction in code size overhead is smaller, because the only a small part of the entire code base has been protected. On average, code size when S-RACFED is implemented is $\times 1.03$ that of the unprotected code, while the code size overhead of RACFED is $\times 1.04$. 


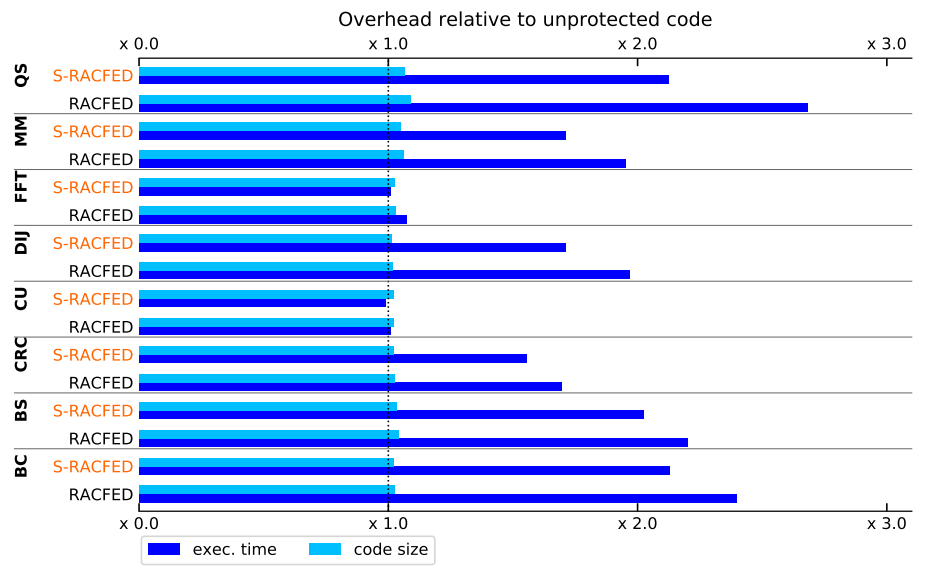

Fig. 3 Execution time overhead of the two CFE detection techniques.

\subsection{Error Detection Latency}

While a lower error detection cost is the advantage of selectively implementing a CFE detection technique, its disadvantage is a higher error detection latency. For the performed experiments, we measured the error detection latency as the number of executed instructions between the point of injection and the execution of the error handler. The results are shown in the box plots of Fig. 4, with the error detection latency data for RACFED shown in dark-blue and the data for S-RACFED depicted in light-blue. The median value for each box plot is shown with the orange line. To show the broad range of data as clear as possible, we chose a logarithmic scale on the $\mathrm{X}$-axis.

As illustrated, the error detection latency for RACFED falls within the range from 1 executed instruction to 30 executed instructions for six out of the eight case studies. For the CU and FFT case studies the upper limit of the range extends to 4000 instructions and 500 instructions, respectively. The median value for most case studies is located around 10 instructions.

The error detection latency of S-RACFED, however, is much higher for all case studies. Here, we can divide the case studies in two groups. The first group consists of BC, CRC, CU and DIJ, and has an error detection latency range from 1 executed instruction to various upper limits, going to 1 million executed instructions before the CFE was detected for the DIJ case study. The second group consists of the remaining case studies and shows little spreading in its error detection latency. For these case studies, the CFE was only detected once the entire program has executed.

These results indicate that S-RACFED is not suited for all types of applications. For example, an application that controls an actuator preferably has a low error detection latency as erroneously controlling an actuator can cause damage or injuries. Applications that are suitable for S-RACFED are applications that process data and 


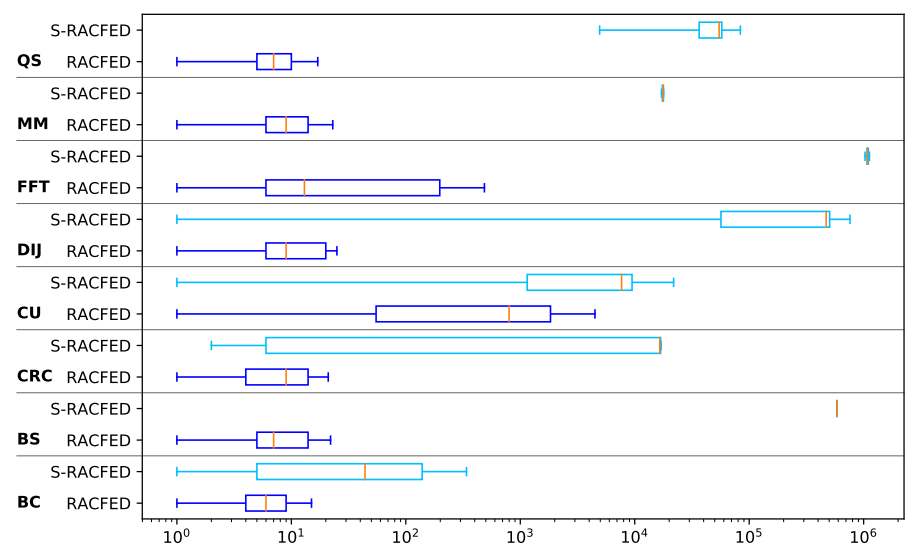

Fig. 4 Boxplots showing the extra error detection latency of S-RACFED compared to RACFED.

return the result. Since only the result is of importance, a higher detection latency can often be tolerated.

\section{Conclusions}

This paper proposed a new approach to selectively implement CFE detection techniques. We propose to only insert signature comparisons and the branches to the error handler in the return basic blocks of the target algorithm. The advantages of this approach are that it is easy to apply and that it can be applied to most, if not all, existing CFE detection techniques.

We validated this approach by applying it to our own RACFED technique to create Selective RACFED (S-RACFED). Next, we performed fault injection experiments for eight case studies and measured the detection ratio, the error detection cost and the error detection latency of S-RACFED. The results show that S-RACFED has approximately the same error detection ratio with a lower overhead when compared to RACFED. This reduction in overhead does come at a cost of a high error detection latency. This high latency makes S-RACFED less suited for actuator controlling applications. Its high detection ratio and low execution time overhead do make S-RACFED very suitable for data processing applications.

Acknowledgements This work is supported by a research grant from the Baekeland program of the Flemish Agency for Innovation and Entrepreneurship (VLAIO) in cooperation with Televic Healthcare NV, under grant agreement IWT 150696. 


\section{References}

1. Alkhalifa, Z., Nair, V.S.S., Krishnamurthy, N., Abraham, J.A.: Design and evaluation of system-level checks for on-line control flow error detection. IEEE Trans. Parallel Distrib. Syst. 10(6), 627-641 (1999). DOI 10.1109/71.774911

2. Asghari, S.A., Abdi, A., Taheri, H., Pedram, H., Pourmozaffari, S.: SEDSR: Soft error detection using software redundancy. Journal of Software Engineering and Applications 5(9), 664-670 (2012). DOI 10.4236/jsea.2012.59078

3. Asghari, S.A., Taheri, H., Pedram, H., Kaynak, O.: Software-based control flow checking against transient faults in industrial environments. IEEE Transactions on Industrial Informatics 10(1), 481-490 (2014). DOI 10.1109/TII.2013.2248373

4. Baffreau, S., Bendhia, S., Ramdani, M., Sicard, E.: Characterisation of microcontroller susceptibility to radio frequency interference. In: Proceedings of the Fourth IEEE International Caracas Conference on Devices, Circuits and Systems (Cat. No.02TH8611), pp. I031-1-I031-5 (2002). DOI 10.1109/ICCDCS.2002.1004088

5. Chielle, E., Rodrigues, G.S., Kastensmidt, F.L., Cuenca-Asensi, S., Tambara, L.A., Rech, P., Quinn, H.: S-SETA: Selective software-only error-detection technique using assertions. IEEE Transactions on Nuclear Science 62(6), 3088-3095 (2015). DOI 10.1109/TNS.2015.2484842

6. Goloubeva, O., Rebaudengo, M., Sonza Reorda, M., Violante, M.: Soft-error detection using control flow assertions. In: Proceedings of the 18th IEEE International Symposium on Defect and Fault Tolerance in VLSI Systems, DFT '03, pp. 581-588. IEEE Computer Society, Washington, DC, USA (2003)

7. Guthaus, M.R., Ringenberg, J.S., Ernst, D., Austin, T.M., Mudge, T., Brown, R.B.: Mibench: A free, commercially representative embedded benchmark suite. In: Proceedings of the Fourth Annual IEEE International Workshop on Workload Characterization. WWC-4 (Cat. No.01EX538), pp. 3-14 (2001). DOI 10.1109/WWC.2001.990739

8. Imperas: Revolutionizing embedded software development. Online (2018). URL http://www.imperas.com/

9. Khudia, D.S., Mahlke, S.: Low cost control flow protection using abstract control signatures. SIGPLAN Not. 48(5), 3-12 (2013). DOI 10.1145/2499369.2465568

10. Li, A., Hong, B.: Software implemented transient fault detection in space computer. Aerospace Science and Technology 11(2), 245 - 252 (2007). DOI 10.1016/j.ast.2006.06.006

11. Nicolescu, B., Savaria, Y., Velazco, R.: SIED: software implemented error detection. In: Proceedings 18th IEEE Symposium on Defect and Fault Tolerance in VLSI Systems, pp. 589-596 (2003)

12. Oh, N., Shirvani, P.P., McCluskey, E.J.: Control-flow checking by software signatures. IEEE Transactions on Reliability 51(1), 111-122 (2002)

13. Sierawski, B.D., Reed, R.A., Mendenhall, M.H., Weller, R.A., Schrimpf, R.D., Wen, S.J., Wong, R., Tam, N., Baumann, R.C.: Effects of scaling on muon-induced soft errors. In: 2011 International Reliability Physics Symposium, pp. 3C.3.1-3C.3.6 (2011). DOI 10.1109/IRPS.2011.5784484

14. Tang, A., Sethumadhavan, S., Stolfo, S.: CLKSCREW: Exposing the perils of securityoblivious energy management. In: 26th USENIX Security Symposium (USENIX Security 17), pp. 1057-1074. USENIX Association, Vancouver, BC (2017)

15. Vankeirsbilck, J., Penneman, N., Hallez, H., Boydens, J.: Random additive control flow error detection. In: B. Gallina, A. Skavhaug, F. Bitsch (eds.) Computer Safety, Reliability, and Security, pp. 220-234. Springer International Publishing, Cham (2018) 